\title{
Maxwellian evolution equations along the uniform optical fiber in Minkowski space
}

\author{
T. Körpinar ${ }^{a}$, R. Cem Demırkol ${ }^{b}$, Z. Körpinar ${ }^{c}$, and V. Asil ${ }^{d}$ \\ ${ }^{a, b}$ Mus Alparslan University, Department of Mathematics, 49250, Muş, Turkey, \\ ${ }^{c}$ Muş Alparslan University, Department of Administration, 49250, Muş, Turkey, \\ ${ }^{d}$ Firat University, Department of Mathematics, 23100, Elazı $\breve{g}$, Turkey.
}

Received 12 March 2020; accepted 16 April 2020

\begin{abstract}
We firstly discuss the geometric phase rotation for an electromagnetic wave traveling along with the optical fiber in Minkowski space. We define two novel types of geometric phases associated with the evolution of the polarization vectors in the normal and binormal directions along with the optical fiber. We also identify the normal-Rytov parallel transportation law and binormal-Rytov parallel transportation law. Moreover, we derive their relationships with the Fermi-Walker transportation law in Minkowski space. Then we solve Maxwell's equations by using geometric quantities associated with the curved path, which characterizes the optical fiber. Finally, we investigate that electromagnetic wave propagation admits the Maxwellian evolution equation for the anholonomic coordinate system in Minkowski space.
\end{abstract}

Keywords: Maxwell's equations; wave propagation; optical fiber; evolution equation; traveling wave hypothesis.

PACS: 03.50.De; 42.25.Ja; 42.65.Tg; 02.40.Hw

\section{Introduction}

Recently, the improvement of the optical fiber technology has led to an enormous interest in wave propagation along twisted waveguides. Especially, rotation effects of the geometric phase of polarized light propagating along the optical fiber have been the research subject of many recent articles regardless of their contents are practical or theoretical. For example, Smith [1] investigated that a rotation of the polarization of light propagating in a monochromatic optical fiber wrapping around the conductor is induced by the magnetic field owing to the electric current flowing. In that study, it was also concluded that the current is proportional to the rotation, which is typical of a few degrees order. The geometric effect of the plane rotation of the light propagation in a mono mode optical fiber tracing a non-planar trajectory was given by Ross [2]. He developed a purely geometric method to calculate the rotation in the helical optical fiber with a constant-torsion. He also supported his results with some measurements on the fiber bent into a helix. Tomita and Chiao [3] generalized the former study of Ross for more general fiber configurations. On the other hand, Chiao and $\mathrm{Wu}$ [4] focused on mainly the theoretical aspect of the effects of geometric phase rotation. They suggested that these effects should be considered as topological features of the traditional Maxwellian theory. Later, Haldane [5] proposed that these effects can be generalized for any arbitrary fiber trajectory without any restriction as in the earlier studies. Apart from previous researches [6-10], in Sec. 3, we propose that an electromagnetic wave acquires novel geometric phases in the normal and binormal directions during its propagation along the curved path in Minkowski space.

In optical fiber researches, light is mostly considered as a carrier of an electromagnetic wave and its features. When it is supposed to propagate within the optical fiber, it is
DOI: https://doi.org/10.31349/RevMexFis.66.431

well-defined, due to the Maxwell's equations. The set of Maxwell's equations implicitly demonstrates how electromagnetic field vectors propagate and explicitly tell sources of the field. In the optical fiber configuration of uniform, isotropic, nonconducting, free-from charge, and nondispersive, we solve Maxwell's equations for the electric and magnetic field vectors. We also derive the Laplacian-like formal equations belonging to the electromagnetic wave propagating along with the fiber in Minkowski space, in which details can be found in $\mathrm{Sec} 4$.

The time evolution of the physical system in a given spacetime structure can be described by the principle of the least action. The evolution of the space curve is a very efficient tool to understand many physical processes such as vortex filaments, dynamics of Heisenberg spin chain, integrable systems, soliton equation theory, sigma models, relativity, water wave theory, fluid dynamics, field theories, linear and nonlinear optics. Evolution systems and equations usually contain intrinsic core geometric meaning. For instance, the sine-Gordon equation, which originally seen in differential geometry, is employed as a model in nonlinear optics, field theories, and dislocation of crystals. The localized induction equation, also known as the Betchov-Da Rios equation or the filament equation, is an idealized example of the evolution of the centerline of a thin vortex tube in a 3D inviscid incompressible fluid $[11,12]$. This equation also constrains the evolution of curves in magnetohydrostatic and steady hydrodynamic problems of nested toroidal flux surfaces [13,14]. The connection between the solutions of the cubic nonlinear Schrodinger equation and the solutions localized induction equation was discovered by Hasimoto [15]. He described a special transformation, including complex curvature and torsion functions of the curve. Furthermore, these functions have been used to define the heat flow, curvature flow, torsion flow, curve shortening flow, and inextensible flow. In all 
these flows, the evolution of geometric quantities is a crucial common element. In Sec. 5, we derive a novel class of curve evolution by considering the propagation of electromagnetic waves and Maxwell's equations along with the optical fiber in Minkowski space. We call this evolution as a Maxwellian evolution of the curve. Then we derive Maxwellian evolution identities of corresponding geometric quantities associated with the given curve. Finally, the paper is completed by stating some important consequences in Sec. 6

\section{Geometric constraints on the space curve in three-dimensional Minkowski space}

In this introductory section, we recall some of the formulae which are used to characterize a three-dimensional vector field and the geometry of curvature and torsion of vector lines in terms of anholonomic coordinates in Minkowski space.
Here, we assume that $\theta=\theta(s, n, b)$ is a space curve lying in a three-dimensional Minkowski space. $s$ is the distance along with the $s$-lines of the curve in the tangential direction, so that unit tangent vector of $s-$ lines is defined by $\overrightarrow{\mathbf{t}}=$ $\overrightarrow{\mathbf{t}}(s, n, b)=(\partial \theta / \partial s) \cdot n$ is the distance along the $n-$ lines of the curve in the normal direction, so that unit tangent vector of $n$-lines is defined by $\overrightarrow{\mathbf{n}}=\overrightarrow{\mathbf{n}}(s, n, b)=(\partial \theta / \partial n) \cdot b$ is the distance along the $b$-lines of the curve in the binormal direction, so that unit tangent vector of $b$-lines is defined by $\overrightarrow{\mathbf{b}}=\overrightarrow{\mathbf{b}}(s, n, b)=(\partial \theta / \partial b)$. The moving trihedron of orthonormal unit vectors $(\overrightarrow{\mathbf{t}}, \overrightarrow{\mathbf{n}}, \overrightarrow{\mathbf{b}})$ provides a platform for investigating the intrinsic features of the curve $\theta$. In this triad, $\overrightarrow{\mathbf{t}}$ is the tangential vector, $\overrightarrow{\mathbf{n}}$ is the normal vector, and $\overrightarrow{\mathbf{b}}$ is the binormal vector of the curve $\theta$. Directional derivatives of the moving trihedron of orthonormal unit vectors $(\overrightarrow{\mathbf{t}}, \overrightarrow{\mathbf{n}}, \overrightarrow{\mathbf{b}})$ can be given by the extended Serret-Frenet relations in the following forms

$$
\begin{aligned}
& \frac{\partial}{\partial s}\left(\begin{array}{c}
\overrightarrow{\mathbf{t}} \\
\overrightarrow{\mathbf{n}} \\
\overrightarrow{\mathbf{b}}
\end{array}\right)=\left(\begin{array}{ccc}
0 & \ell_{2} \kappa & 0 \\
-\ell_{1} \kappa & 0 & -\ell_{3} \tau \\
0 & \ell_{2} \tau & 0
\end{array}\right)\left(\begin{array}{c}
\overrightarrow{\mathbf{t}} \\
\overrightarrow{\mathbf{n}} \\
\overrightarrow{\mathbf{b}}
\end{array}\right), \\
& \frac{\partial}{\partial n}\left(\begin{array}{c}
\overrightarrow{\mathbf{t}} \\
\overrightarrow{\mathbf{n}} \\
\overrightarrow{\mathbf{b}}
\end{array}\right)=\left(\begin{array}{ccc}
0 & \ell_{2} \delta_{n s} & \ell_{3}\left(\ell_{1} \pi_{b}-\tau\right) \\
-\ell_{2} \delta_{n s} & 0 & -\ell_{2} \ell_{3} \operatorname{div} \overrightarrow{\mathbf{b}} \\
-\ell_{1}\left(\ell_{1} \pi_{b}-\tau\right) & \operatorname{div} \overrightarrow{\mathbf{b}} & 0
\end{array}\right)\left(\begin{array}{c}
\overrightarrow{\mathbf{t}} \\
\overrightarrow{\mathbf{n}} \\
\overrightarrow{\mathbf{b}}
\end{array}\right) \\
& \frac{\partial}{\partial b}\left(\begin{array}{c}
\overrightarrow{\mathbf{t}} \\
\overrightarrow{\mathbf{n}} \\
\overrightarrow{\mathbf{b}}
\end{array}\right)=\left(\begin{array}{ccc}
0 & -\ell_{2}\left(\ell_{1} \pi_{n}-\tau\right) & \ell_{3} \delta_{b s} \\
\ell_{1}\left(\ell_{1} \pi_{n}-\tau\right) & 0 & \ell_{1} \kappa+\operatorname{div} \overrightarrow{\mathbf{n}} \\
-\ell_{1} \delta_{b s} & \ell_{1}\left(\ell_{1} \kappa+\operatorname{div} \overrightarrow{\mathbf{n}}\right) & 0
\end{array}\right)\left(\begin{array}{c}
\overrightarrow{\mathbf{t}} \\
\overrightarrow{\mathbf{n}} \\
\overrightarrow{\mathbf{b}}
\end{array}\right)
\end{aligned}
$$

The inner product and the cross product are defined by

$$
\begin{aligned}
\ell_{1} & =\overrightarrow{\mathbf{t}} \cdot \overrightarrow{\mathbf{t}}, \ell_{2}=\overrightarrow{\mathbf{n}} \cdot \overrightarrow{\mathbf{n}}, \ell_{3}=\overrightarrow{\mathbf{b}} \cdot \overrightarrow{\mathbf{b}} \\
\overrightarrow{\mathbf{t}} \times \overrightarrow{\mathbf{n}} & =\ell_{3} \overrightarrow{\mathbf{b}}, \overrightarrow{\mathbf{n}} \times \overrightarrow{\mathbf{b}}=\ell_{1} \overrightarrow{\mathbf{t}}, \overrightarrow{\mathbf{b}} \times \overrightarrow{\mathbf{t}}=\ell_{2} \overrightarrow{\mathbf{n}} .
\end{aligned}
$$

The gradient operator $\nabla$ is expressed by

$$
\nabla=\ell_{1} \overrightarrow{\mathbf{t}} \frac{\partial}{\partial s}+\ell_{2} \overrightarrow{\mathbf{n}} \frac{\partial}{\partial n}+\ell_{3} \overrightarrow{\mathbf{b}} \frac{\partial}{\partial b},
$$

and $\delta_{n s}=\overrightarrow{\mathbf{n}} \cdot(\partial / \partial n) \overrightarrow{\mathbf{t}}$, and $\delta_{b s}=\overrightarrow{\mathbf{b}} \cdot(\partial / \partial b) \overrightarrow{\mathbf{t}}$ [14] Thus, other geometric quantities are given by

$$
\begin{aligned}
\operatorname{div} \overrightarrow{\mathbf{t}} & =\ell_{2} \delta_{n s}+\ell_{3} \delta_{b s}, \\
\operatorname{div} \overrightarrow{\mathbf{n}} & =-\ell_{1} \kappa+\ell_{3} \overrightarrow{\mathbf{b}} \cdot \frac{\partial}{\partial b} \overrightarrow{\mathbf{n}}, \\
\operatorname{div} \overrightarrow{\mathbf{b}} & =-\ell_{2} \overrightarrow{\mathbf{b}} \cdot \frac{\partial}{\partial n} \overrightarrow{\mathbf{n}}, \\
\operatorname{curl} \overrightarrow{\mathbf{t}} & =\ell_{1} \pi_{s} \overrightarrow{\mathbf{t}}+\ell_{1} \kappa \overrightarrow{\mathbf{b}}, \\
\operatorname{curl} \overrightarrow{\mathbf{n}} & =\ell_{1} \ell_{3}(\operatorname{div} \overrightarrow{\mathbf{b}}) \overrightarrow{\mathbf{t}}+\ell_{2} \pi_{n} \overrightarrow{\mathbf{n}}+\ell_{1} \delta_{n s} \overrightarrow{\mathbf{b}}, \\
\operatorname{curl} \overrightarrow{\mathbf{b}} & =-\ell_{2}\left(\kappa+\ell_{1} \operatorname{div} \overrightarrow{\mathbf{n}}\right) \overrightarrow{\mathbf{t}}-\ell_{1} \delta_{b s} \overrightarrow{\mathbf{n}}+\ell_{3} \pi_{b} \overrightarrow{\mathbf{b}}
\end{aligned}
$$

The abnormalies of the $\overrightarrow{\mathbf{t}}-$ field, $\overrightarrow{\mathbf{n}}-$ field, and $\overrightarrow{\mathbf{b}}-$ field are respectively computed by

$$
\begin{aligned}
& \pi_{s}=\overrightarrow{\mathbf{t}} \cdot \operatorname{curl} \overrightarrow{\mathbf{t}}=\ell_{1} \ell_{2} \ell_{3}\left(-\overrightarrow{\mathbf{b}} \cdot \frac{\partial}{\partial n} \overrightarrow{\mathbf{t}}+\overrightarrow{\mathbf{n}} \cdot \frac{\partial}{\partial b} \overrightarrow{\mathbf{t}}\right) \\
& \pi_{n}=\overrightarrow{\mathbf{n}} \cdot \operatorname{curl} \overrightarrow{\mathbf{n}}=\ell_{1}\left(\overrightarrow{\mathbf{t}} \cdot \frac{\partial}{\partial b} \overrightarrow{\mathbf{n}}+\tau\right) \\
& \pi_{b}=\overrightarrow{\mathbf{b}} \cdot \operatorname{curl} \overrightarrow{\mathbf{b}}=\ell_{1}\left(\tau-\overrightarrow{\mathbf{t}} \cdot \frac{\partial}{\partial n} \overrightarrow{\mathbf{b}}\right)
\end{aligned}
$$

\section{Geometric phases of the electromagnetic waves traveling in the $(\vec{t}, \overrightarrow{\mathbf{n}}, \overrightarrow{\mathrm{b}})$ direction along the optical fiber in Minkowski space}

Let us consider the propagation of linearly polarized light along with an ideal mono mode optical fiber in Minkowski space. The ideality of the fiber implies that the fiber has no elastoptic effects such as torsional-stress-induced circular birefringence and bend-induced linear birefringence. 
An electromagnetic wave in the optical fiber can consist of the propagation of light, which travels in the optical fiber and oscillates in time. Thus, naively speaking, we assume for the rest of the paper that the electromagnetic wave propagates along with the optical fiber in which its axis is given by the curve $\theta(s, n, b)$ in Minkowski space. Owing to the vectorial nature of the light, it is an obvious fact that electromagnetic waves can be described by using the adapted coordinate frame and associated vector fields.

The orientation of the propagation and polarization of the electromagnetic wave in the fiber can be defined by the orthonormal set of vectors $(\overrightarrow{\mathbf{t}}, \overrightarrow{\mathbf{n}}, \overrightarrow{\mathbf{b}})$. The electromagnetic wave propagation is in the direction of $\overrightarrow{\mathbf{t}}=\overrightarrow{\mathbf{t}}(s, n, b)$, and the polarization of the electromagnetic wave is referred by the direction of the electric field vector $\overrightarrow{\mathbf{E}}=\overrightarrow{\mathbf{E}}(s, n, b)$. Since light propagates as an electromagnetic wave inside the optical fiber the electromagnetic wave also carries inherently magnetic field vector $\overrightarrow{\mathbf{B}}=\overrightarrow{\mathbf{B}}(s, n, b)$. As a consequence, the electromagnetic wave vectors $(\overrightarrow{\mathbf{t}}, \overrightarrow{\mathbf{E}}, \overrightarrow{\mathbf{B}})$ can be considered as a physically observable adapted coordinate frame, which is expressed in terms of Frenet-Serret orthonormal unit vectors $(\overrightarrow{\mathbf{t}}, \overrightarrow{\mathbf{n}}, \overrightarrow{\mathbf{b}})$.

When the electromagnetic wave is constrained to travel along with a space curve, the curve geometry provides to relate the geometric phase and the rotation of the polarization of the electromagnetic wave vectors. Many research effort has been devoted to achieving to explore this connection and further details. However, all these efforts have been made by only considering the light ray propagating along with the optical fiber described by the curve, which is parameterized by only the $s$ parameter. According to this approach, for an electromagnetic wave having the form of a space curve, the electric field vector $\overrightarrow{\mathbf{E}}$ and the magnetic field vector $\overrightarrow{\mathbf{B}}$ are supposed to perform a rotation in the tangential direction concerning to the orthonormal unit vectors of Frenet-Serret's triple $(\overrightarrow{\mathbf{t}}, \overrightarrow{\mathbf{n}}, \overrightarrow{\mathbf{b}})$. Then the parallel transportation law of polarization vectors $\overrightarrow{\mathbf{E}}$ and $\overrightarrow{\mathbf{B}}$ in the tangential direction along the optical fiber is verified by the Rytov law in the following way $[6,10,16]$

$$
\begin{aligned}
& \overrightarrow{\mathbf{E}}_{s}= \pm\left(\overrightarrow{\mathbf{E}} \cdot \overrightarrow{\mathbf{t}_{s}}\right) \overrightarrow{\mathbf{t}} \\
& \overrightarrow{\mathbf{B}}_{s}= \pm\left(\overrightarrow{\mathbf{B}} \cdot \overrightarrow{\mathbf{t}_{s}}\right) \overrightarrow{\mathbf{t}}
\end{aligned}
$$

Here, \pm sign occurs depending on the variable character of the tangent vector of the curve $\theta$. This geometric transportation law implies that polarization vectors $\overrightarrow{\mathbf{E}}$ and $\overrightarrow{\mathbf{B}}$ do not rotate around the tangent vector $\overrightarrow{\mathbf{t}}$ in the tangential direction however, they rotate with respect to the osculating plane, which is spanned by the $(\overrightarrow{\mathbf{n}}, \overrightarrow{\mathbf{b}})$ basis, in the tangential direction in Minkowski space. Consequently, a geometric phase $\rho=\rho(s, n, b)$ in the tangential direction is defined by the rotation of polarization vectors $\overrightarrow{\mathbf{E}}$ and $\overrightarrow{\mathbf{B}}$ concerning $(\overrightarrow{\mathbf{n}}, \overrightarrow{\mathbf{b}})$ with an angular velocity

$$
\frac{\partial}{\partial s} \rho=\tau(s) .
$$

In this section, we demonstrate that the electromagnetic wave acquires a novel class of geometric phases in the normal and binormal directions during the propagation of the electromagnetic wave along the curved path in Minkowski space. These novel geometric phases are described by the geometric quantities, which are induced by the characterization of the curve in the three-dimensional Minkowski space. We also show that the parallel transportation law of polarization vectors $\overrightarrow{\mathbf{E}}$ and $\overrightarrow{\mathbf{B}}$ in the normal and binormal directions along the curved path is also related to another geometric concept known as Fermi-Walker transportation. Thus, we obtain corresponding Fermi-Walker transportation definition, which is the counterpart of the Rytov law, for the polarization vectors $\overrightarrow{\mathbf{E}}$ and $\overrightarrow{\mathbf{B}}$ in the normal and binormal directions in Minkowski space.

Case 1. Let the polarization vectors of the propagated electromagnetic wave are referred by the electric field vector $\overrightarrow{\mathbf{E}}=\overrightarrow{\mathbf{E}}(s, n, b)$ and magnetic field vector $\overrightarrow{\mathbf{B}}=$ $\overrightarrow{\mathbf{B}}(s, n, b)$. They are also both perpendicular to the propagation vector $\overrightarrow{\mathbf{t}}=\overrightarrow{\mathbf{t}}(s, n, b)$ along with the optical fiber in Minkowski space. The change of the electric vector $\overrightarrow{\mathbf{E}}$ between any two points in the normal direction along with the curved path $\theta$ is given by

$$
\frac{\partial}{\partial n} \overrightarrow{\mathbf{E}}(s, n, b)=\overrightarrow{\mathbf{E}}_{n}=\varpi \overrightarrow{\mathbf{t}}+\lambda \overrightarrow{\mathbf{n}}+\gamma \overrightarrow{\mathbf{b}},
$$

where $\varpi=\varpi(s, n, b), \lambda=\lambda(s, n, b)$, and $\gamma=\gamma(s, n, b)$ are sufficiently smooth arbitrary functions along the $\theta$. If we use the fact that $\overrightarrow{\mathbf{t}} \cdot \overrightarrow{\mathbf{E}}=0$ and $\overrightarrow{\mathbf{E}} \cdot \overrightarrow{\mathbf{E}}=C$, where $C$ is a constant term, then we have

$$
\begin{aligned}
\frac{\partial}{\partial n} \overrightarrow{\mathbf{t}} \cdot \overrightarrow{\mathbf{E}} & =-\overrightarrow{\mathbf{t}} \cdot \frac{\partial}{\partial n} \overrightarrow{\mathbf{E}}, \\
\frac{\partial}{\partial n} \overrightarrow{\mathbf{E}} \cdot \overrightarrow{\mathbf{E}} & =0 .
\end{aligned}
$$

Thus, from Eqs. $(2,17-19)$, it is obtained that

$$
\overrightarrow{\mathbf{E}}_{n}=\left(\ell_{3} \delta_{n s} \mathbf{E}^{n}+\ell_{2}\left(\ell_{1} \pi_{b}-\tau\right) \mathbf{E}^{b}\right) \overrightarrow{\mathbf{t}}+\eta(\overrightarrow{\mathbf{E}} \times \overrightarrow{\mathbf{t}}),
$$

where $\eta$ is a constant term and independent of $\overrightarrow{\mathbf{E}}$. Here, we also use the fact that $\ell_{3}=-\ell_{1} \ell_{2}$, where $\ell_{i}= \pm 1$ for $i=1,2,3$. This last equation is the most general form of the variation of the electric field vector in the normal direction along with the fiber in Minkowski space. The last term of the Eq. (20) determines the rotation of the electric field $\overrightarrow{\mathbf{E}}$ in the normal direction around the $\overrightarrow{\mathrm{t}}$. We should also note that the above expression was obtained without considering the geometric optics approximation. Hence, we can assume that $\eta=0$ since the optical fiber does not favor the left or right rotation of the field. As a result, we can conclude that the electric field $\overrightarrow{\mathbf{E}}$ is parallel transported in the normal direction along with the fiber since it satisfies the following modified 
type of Rytov parallel transportation law in Minkowski space

$$
\begin{aligned}
& \overrightarrow{\mathbf{E}}_{n}=\left(\ell_{3} \delta_{n s} \mathbf{E}^{n}+\ell_{2}\left(\ell_{1} \pi_{b}-\tau\right) \mathbf{E}^{b}\right) \overrightarrow{\mathbf{t}} \\
& \overrightarrow{\mathbf{E}}_{n}=-\ell_{1}\left(\overrightarrow{\mathbf{E}} \cdot \overrightarrow{\mathbf{t}_{n}}\right) \overrightarrow{\mathbf{t}}
\end{aligned}
$$

Here, we call the expression in the Eq. (22) as a normalRytov parallel transportation law in Minkowski space. Thus, in Minkowski space, we can easily see that normal-Rytov parallel transported law and Fermi-Walker parallel transported law in the normal direction for the electric field $\overrightarrow{\mathbf{E}}$ is associated with each other. Consequently, we may state that $\overrightarrow{\mathbf{E}}$ is normal-Rytov parallel transported if and only if it is Fermi-Walker parallel transported in the normal direction in Minkowski space. In this space, the definition of the FermiWalker derivative in the normal direction is defined by

$$
\overrightarrow{\mathbf{A}}_{n}^{\mathcal{F W}}=\overrightarrow{\mathbf{A}}_{n}+\ell_{1}\left(\overrightarrow{\mathbf{A}} \cdot \overrightarrow{\mathbf{t}_{n}}\right) \overrightarrow{\mathbf{t}}-(\overrightarrow{\mathbf{A}} \cdot \overrightarrow{\mathbf{t}}) \overrightarrow{\mathbf{t}_{n}}
$$

where $\overrightarrow{\mathbf{A}}$ is an arbitrary vector field along with the curve. The proof is left to the reader since it is obvious from Eqs. $(2,18$ 23).

Now, we examine a very important consequence of the choice of the parallel transportation of the electric field vector $\overrightarrow{\mathbf{E}}$ in the normal direction along with the optical fiber in Minkowski space. A natural choice for the selection of the electric field $\overrightarrow{\mathbf{E}}$ can be made by

$$
\overrightarrow{\mathbf{E}}=\ell_{1} \mathbf{E}^{n} \overrightarrow{\mathbf{n}}+\ell_{3} \mathbf{E}^{b} \overrightarrow{\mathbf{b}}
$$

where $\mathbf{E}^{n}, \mathbf{E}^{b}$ are arbitrarily smooth components of the $\overrightarrow{\mathbf{n}}$ and $\vec{b}$ vectors, respectively. The derivative of the electric field $\overrightarrow{\mathbf{E}}$ in the normal direction yields that

$$
\begin{gathered}
\frac{\partial}{\partial n} \overrightarrow{\mathbf{E}}(s, n, b)=\overrightarrow{\mathbf{E}}_{n}=\left(\ell_{3} \delta_{n s} \mathbf{E}^{n}+\ell_{2}\left(\ell_{1} \pi_{b}-\tau\right) \mathbf{E}^{b}\right) \overrightarrow{\mathbf{t}} \\
\quad+\left(\ell_{1} \mathbf{E}_{n}^{n}+\ell_{3} \mathbf{E}^{b} \operatorname{div} \overrightarrow{\mathbf{b}}\right) \overrightarrow{\mathbf{n}}+\left(\ell_{3} \mathbf{E}_{n}^{b}+\mathbf{E}^{n} \operatorname{div} \overrightarrow{\mathbf{b}}\right) \overrightarrow{\mathbf{b}}
\end{gathered}
$$

If $\overrightarrow{\mathbf{E}}$ is assumed to be parallel transported in the normal direction along with the fiber, then comparing the Eq. (22) and Eq. (25) implies that

$$
\frac{\partial}{\partial n}\left(\begin{array}{c}
\mathbf{E}^{n} \\
\mathbf{E}^{b}
\end{array}\right)=\left(\begin{array}{cc}
0 & \ell_{2} \operatorname{div} \overrightarrow{\mathbf{b}} \\
-\ell_{3} \operatorname{div} \overrightarrow{\mathbf{b}} & 0
\end{array}\right)\left(\begin{array}{l}
\mathbf{E}^{n} \\
\mathbf{E}^{b}
\end{array}\right) .
$$

Hence, we can conclude that Eq. (26) defines the rotation of the polarization plane in the normal direction so that a geometric phase $\rho=\rho(s, n, b)$ in the normal direction is described by

$$
\frac{\partial}{\partial n} \rho=\operatorname{div} \overrightarrow{\mathbf{b}} \text {. }
$$

We can also characterize the other polarization vector $\overrightarrow{\mathbf{B}}\left(\overrightarrow{\mathbf{B}}=\ell_{1} \mathbf{B}^{n} \overrightarrow{\mathbf{n}}+\ell_{3} \mathbf{B}^{b} \overrightarrow{\mathbf{b}}\right)$ because $\overrightarrow{\mathbf{B}}=\overrightarrow{\mathbf{t}} \times \overrightarrow{\mathbf{E}}$. That is, we can express the magnetic field vector in terms of the components of the $\overrightarrow{\mathbf{E}}$ in the following form.

$$
\overrightarrow{\mathbf{B}}=\ell_{1} \mathbf{E}^{b} \overrightarrow{\mathbf{n}}-\ell_{2} \mathbf{E}^{n} \overrightarrow{\mathbf{b}}
$$

where $\mathbf{B}^{n}=\mathbf{E}^{b}$ and $\mathbf{B}^{b}=\ell_{1} \mathbf{E}^{n}$. By using the fact that $\overrightarrow{\mathbf{B}} \perp \overrightarrow{\mathbf{E}}$ and $\overrightarrow{\mathbf{B}} \perp \overrightarrow{\mathbf{t}}$, we compute that

$$
\begin{aligned}
& \frac{\partial}{\partial n} \overrightarrow{\mathbf{B}}(s, n, b)=\overrightarrow{\mathbf{B}}_{n}=\left(\ell_{3} \delta_{n s} \mathbf{E}^{b}-\ell_{3}\left(\ell_{1} \pi_{b}-\tau\right) \mathbf{E}^{n}\right) \overrightarrow{\mathbf{t}} \\
& \quad+\left(\ell_{1} \mathbf{E}_{n}^{b}-\ell_{2} \mathbf{E}^{n} \operatorname{div} \overrightarrow{\mathbf{b}}\right) \overrightarrow{\mathbf{n}}+\left(-\ell_{2} \mathbf{E}_{n}^{n}+\mathbf{E}^{b} \operatorname{div} \overrightarrow{\mathbf{b}}\right) \overrightarrow{\mathbf{b}}
\end{aligned}
$$

which satisfies

$$
\begin{aligned}
& \overrightarrow{\mathbf{B}}_{n} \cdot \overrightarrow{\mathbf{E}}=-\overrightarrow{\mathbf{B}} \cdot \overrightarrow{\mathbf{E}}_{n}, \\
& \overrightarrow{\mathbf{B}}_{n} \cdot \overrightarrow{\mathbf{t}}=-\overrightarrow{\mathbf{B}} \cdot \overrightarrow{\mathbf{t}_{n}}
\end{aligned}
$$

If we check Eqs. $(32,33)$, then it is obtained that $\ell_{1}=\ell_{2}=1$ and $\ell_{3}=-1$, which implies that the optical fiber coupling into the curve path $\theta$ is represented by the spacelike curve having a timelike binormal in the normal direction in Minkowski space. Moreover, if we multiply both sides of the Eq. (30) by $\overrightarrow{\mathbf{t}}$ then it can be verified that $\overrightarrow{\mathbf{B}}$ also satisfies the normal-Rytov law with acquiring the same geometric phase as the electric field $\overrightarrow{\mathbf{E}}$ in Minkowski space.

$$
\overrightarrow{\mathbf{B}}_{n}=-\ell_{1}\left(\overrightarrow{\mathbf{B}} \cdot \overrightarrow{\mathbf{t}_{n}}\right) \overrightarrow{\mathbf{t}}
$$

Case 2. Let the polarization vectors of the propagated electromagnetic wave are referred by the electric field vector $\overrightarrow{\mathbf{E}}=\overrightarrow{\mathbf{E}}(s, n, b)$ and magnetic field vector $\overrightarrow{\mathbf{B}}=\overrightarrow{\mathbf{B}}(s, n, b)$. They are also both perpendicular to the propagation vector $\overrightarrow{\mathbf{t}}=\overrightarrow{\mathbf{t}}(s, n, b)$ along with the optical fiber in Minkowski space. The change of the electric vector $\overrightarrow{\mathbf{E}}$ between any two points in the binormal direction along with the curved path $\theta$ is given by

$$
\frac{\partial}{\partial b} \overrightarrow{\mathbf{E}}(s, n, b)=\overrightarrow{\mathbf{E}}_{b}=\varpi^{\circ} \overrightarrow{\mathbf{t}}+\lambda^{\circ} \overrightarrow{\mathbf{n}}+\gamma^{\circ} \overrightarrow{\mathbf{b}}
$$

where $\varpi^{\circ}=\varpi^{\circ}(s, n, b), \lambda^{\circ}=\lambda^{\circ}(s, n, b)$, and $\gamma^{\circ}=$ $\gamma^{\circ}(s, n, b)$ are sufficiently smooth arbitrary functions. If we use the fact that $\overrightarrow{\mathbf{t}} \cdot \overrightarrow{\mathbf{E}}=0$ and $\overrightarrow{\mathbf{E}} \cdot \overrightarrow{\mathbf{E}}=C^{\circ}$, where $C^{\circ}$ is a constant term, then we have

$$
\begin{aligned}
& \frac{\partial}{\partial b} \overrightarrow{\mathbf{t}} \cdot \overrightarrow{\mathbf{E}}=-\overrightarrow{\mathbf{t}} \cdot \frac{\partial}{\partial b} \overrightarrow{\mathbf{E}}, \\
& \frac{\partial}{\partial b} \overrightarrow{\mathbf{E}} \cdot \overrightarrow{\mathbf{E}}=0 .
\end{aligned}
$$

Thus, from Eqs. $(3,32-34)$ it is obtained that

$$
\overrightarrow{\mathbf{E}}_{b}=\left(-\ell_{3}\left(\pi_{n}-\tau\right) \mathbf{E}^{n}+\ell_{2} \delta_{b s} \mathbf{E}^{b}\right) \overrightarrow{\mathbf{t}}+\eta^{\circ}(\overrightarrow{\mathbf{E}} \times \overrightarrow{\mathbf{t}}),
$$

where $\eta^{\circ}$ is a constant term and independent of $\overrightarrow{\mathbf{E}}$. This last equation is the most general form of the variation of the electric field vector in the binormal direction along with the fiber in Minkowski space. The last term of the Eq. (35) determines the rotation of the electric field $\overrightarrow{\mathbf{E}}$ in the binormal direction around the $\overrightarrow{\mathbf{t}}$. Here we should also note that the above expression was obtained without considering the geometric optics approximation again. Hence, we can assume that $\eta^{\circ}=0$ 
since the optical fiber does not favor the left or right rotation of the field. As a result, we can conclude that the electric field $\overrightarrow{\mathbf{E}}$ is parallel transported in the binormal direction along with the fiber since it satisfies the following modified type of Rytov parallel transportation law in Minkowski space

$$
\begin{aligned}
& \overrightarrow{\mathbf{E}}_{b}=\left(-\ell_{3}\left(\pi_{n}-\tau\right) \mathbf{E}^{n}+\ell_{2} \delta_{b s} \mathbf{E}^{b}\right) \overrightarrow{\mathbf{t}}, \\
& \overrightarrow{\mathbf{E}}_{b}=-\left(\overrightarrow{\mathbf{E}} \cdot \overrightarrow{\mathbf{t}_{b}}\right) \overrightarrow{\mathbf{t}} .
\end{aligned}
$$

Here we call the expression in the Eq. (37) as a binormalRytov parallel transportation law in Minkowski space. Thus, in Minkowski space, we can easily see that binormal-Rytov parallel transported law and Fermi-Walker parallel transported law in the binormal direction for the electric field $\overrightarrow{\mathbf{E}}$ is associated with each other. Consequently, we may state that $\overrightarrow{\mathbf{E}}$ is binormal-Rytov parallel transported if and only if it is Fermi-Walker parallel transported in the binormal direction in Minkowski space. In this space, the definition of the Fermi-Walker derivative in the binormal direction is defined by

$$
\overrightarrow{\mathbf{A}}_{b}^{\mathcal{F W}}=\overrightarrow{\mathbf{A}}_{b}+\ell_{1}\left(\overrightarrow{\mathbf{A}} \cdot \overrightarrow{\mathbf{t}_{n}}\right) \overrightarrow{\mathbf{t}}-(\overrightarrow{\mathbf{A}} \cdot \overrightarrow{\mathbf{t}}) \overrightarrow{\mathbf{t}_{b}}
$$

where $\overrightarrow{\mathbf{A}}$ is an arbitrary vector field along with the curve. Thus, one can easily see that binormal-Rytov parallel transported law and Fermi-Walker parallel transported law in the binormal direction of the electric field $\overrightarrow{\mathbf{E}}$ is interchangeable with each other. The proof is left to the reader since it is obvious from Eqs. (3,33-38).

Now, we examine a very important consequence of the choice of the parallel transportation of the electric field vector $\overrightarrow{\mathbf{E}}$ in the binormal direction along with the optical fiber in Minkowski space. A natural choice for the selection of the electric field $\overrightarrow{\mathbf{E}}$ can be made by

$$
\overrightarrow{\mathbf{E}}=\ell_{1} \mathbf{E}^{n} \overrightarrow{\mathbf{n}}+\ell_{3} \mathbf{E}^{b} \overrightarrow{\mathbf{b}}
$$

where $\mathbf{E}^{n}, \mathbf{E}^{b}$ are arbitrarily smooth components of the $\overrightarrow{\mathbf{n}}$ and $\vec{b}$ vectors, respectively. The derivative of the electric field $\overrightarrow{\mathbf{E}}$ in the binormal direction yields that

$$
\begin{aligned}
\frac{\partial}{\partial b} \overrightarrow{\mathbf{E}}(s, n, b) & =\overrightarrow{\mathbf{E}}_{b}=\left(\left(\pi_{n}-\tau\right) \mathbf{E}^{n}+\ell_{2} \delta_{b s} \mathbf{E}^{b}\right) \overrightarrow{\mathbf{t}} \\
& +\left(\ell_{1} \mathbf{E}_{b}^{n}-\ell_{2} \mathbf{E}^{b}\left(\ell_{1} \kappa+\operatorname{div} \overrightarrow{\mathbf{n}}\right)\right) \overrightarrow{\mathbf{n}} \\
& +\left(\ell_{3} \mathbf{E}_{b}^{b}+\ell_{1} \mathbf{E}^{n}\left(\ell_{1} \kappa+\operatorname{div} \overrightarrow{\mathbf{n}}\right)\right) \overrightarrow{\mathbf{b}}
\end{aligned}
$$

If $\overrightarrow{\mathbf{E}}$ is assumed to be parallel transported in the binormal direction along with the fiber, then comparing the Eq. (37) and Eq. (40) implies that

$\frac{\partial}{\partial b}\left(\begin{array}{l}\mathbf{E}^{n} \\ \mathbf{E}^{b}\end{array}\right)=\left(\begin{array}{cc}0 & -\ell_{3}(\kappa+\operatorname{div} \overrightarrow{\mathbf{n}}) \\ \ell_{2}(\kappa+\operatorname{div} \overrightarrow{\mathbf{n}}) & 0\end{array}\right)\left(\begin{array}{l}\mathbf{E}^{n} \\ \mathbf{E}^{b}\end{array}\right)$.

Here if we also compare the Eq. (40) and Eqs. $(36,37)$ it yields $\ell_{1}=\ell_{2}=1$ and $\ell_{3}=-1$, which implies that the curved path $\theta$ representing the path of the optical fiber $\theta$ found to be a spacelike curve having a timelike binormal in the binormal direction in Minkowski space. We can further conclude that Eq. (41) defines the rotation of the polarization plane in the binormal direction so that a geometric phase $\rho=\rho(s, n, b)$ in the binormal direction is described by

$$
\frac{\partial}{\partial b} \rho=\kappa+\operatorname{div} \overrightarrow{\mathbf{n}} .
$$

One can also characterize the other polarization vector $\overrightarrow{\mathbf{B}}\left(\overrightarrow{\mathbf{B}}=\ell_{1} \mathbf{B}^{n} \overrightarrow{\mathbf{n}}+\ell_{3} \mathbf{B}^{b} \overrightarrow{\mathbf{b}}\right)$ because $\overrightarrow{\mathbf{B}}=\overrightarrow{\mathbf{t}} \times \overrightarrow{\mathbf{E}}$. That is, we can express the magnetic field vector in terms of the components of the $\overrightarrow{\mathbf{E}}$ in the following form

$$
\overrightarrow{\mathbf{B}}=\mathbf{E}^{b} \overrightarrow{\mathbf{n}}-\mathbf{E}^{n} \overrightarrow{\mathbf{b}},
$$

where $\mathbf{B}^{n}=\mathbf{E}^{b}$ and $\mathbf{B}^{b}=\mathbf{E}^{n}$. By using the fact that $\overrightarrow{\mathbf{B}} \perp \overrightarrow{\mathbf{E}}$ and $\overrightarrow{\mathbf{B}} \perp \overrightarrow{\mathbf{t}}$, we compute that

$$
\begin{aligned}
\frac{\partial}{\partial b} \overrightarrow{\mathbf{B}}(s, n, b) & =\overrightarrow{\mathbf{B}}_{b}=\left(\left(\pi_{n}-\tau\right) \mathbf{E}^{b}+\delta_{b s} \mathbf{E}^{n}\right) \overrightarrow{\mathbf{t}} \\
& +\left(\mathbf{E}_{b}^{b}-\mathbf{E}^{n}(\kappa+\operatorname{div} \overrightarrow{\mathbf{n}})\right) \overrightarrow{\mathbf{n}}+\left(-\mathbf{E}_{b}^{n}\right. \\
& \left.+\mathbf{E}^{b}(\kappa+\operatorname{div} \overrightarrow{\mathbf{n}})\right) \overrightarrow{\mathbf{b}},
\end{aligned}
$$

which satisfies

$$
\begin{aligned}
& \overrightarrow{\mathbf{B}}_{b} \cdot \overrightarrow{\mathbf{E}}=-\overrightarrow{\mathbf{B}} \cdot \overrightarrow{\mathbf{E}}_{b}, \\
& \overrightarrow{\mathbf{B}}_{b} \cdot \overrightarrow{\mathbf{t}}=-\overrightarrow{\mathbf{B}} \cdot \overrightarrow{\mathbf{t}}_{b}
\end{aligned}
$$

So, if we multiply both sides of the Eq. (45) by $\overrightarrow{\mathbf{t}}$, then it can be verified that $\overrightarrow{\mathbf{B}}$ also satisfies the binormal-Rytov law with acquiring the same geometric phase as the electric field $\overrightarrow{\mathbf{E}}$ in Minkowski space and we finally obtain

$$
\overrightarrow{\mathbf{B}}_{b}=-\left(\overrightarrow{\mathbf{B}} \cdot \overrightarrow{\mathbf{t}_{n}}\right) \overrightarrow{\mathbf{t}}
$$

\section{Maxwell's equations for electromagnetic waves propagating along the optical fiber in Minkowski space}

Maxwell's equation has an important role to understand the electromagnetic theory. It provides an exact comprehension and observation of the propagation of light along with the optical fiber. Electromagnetic waves propagated along the optical fiber satisfy the following conditions supposing that the fiber is uniform, isotropic, nonconducting, free-from charge, and non-dispersive [17]

$$
\begin{aligned}
\nabla \cdot \overrightarrow{\mathbf{E}} & =0, \\
\nabla \cdot \overrightarrow{\mathbf{B}} & =0, \\
\nabla \times \overrightarrow{\mathbf{B}} & =\epsilon v \frac{\partial \overrightarrow{\mathbf{E}}}{\partial u}, \\
\nabla \times \overrightarrow{\mathbf{E}} & =-\frac{\partial \overrightarrow{\mathbf{B}}}{\partial u} .
\end{aligned}
$$


where $\epsilon$ and $v$ have the same values at all points, and they do not depend on the direction of propagation since they are not functions of frequency. In the previous section, we have already investigated that the curved path $\theta$, which characterizes the geometry of the optical fiber, is a spacelike curve having a timelike binormal both in the normal and binormal directions. Hence, for the rest of the paper, it is assumed that $\ell_{1}=\ell_{2}=1$ and $\ell_{3}=-1$. From Eqs. $(14,25,40,47)$, we compute that

$$
\begin{aligned}
& 0=\nabla \cdot \overrightarrow{\mathbf{E}}=\left(\overrightarrow{\mathbf{t}} \frac{\partial}{\partial s}+\overrightarrow{\mathbf{n}} \frac{\partial}{\partial n}-\overrightarrow{\mathbf{b}} \frac{\partial}{\partial b}\right) \cdot \overrightarrow{\mathbf{E}}, \\
& 0=\overrightarrow{\mathbf{t}} \cdot \frac{\partial}{\partial s} \overrightarrow{\mathbf{E}}+\overrightarrow{\mathbf{n}} \cdot \frac{\partial}{\partial n} \overrightarrow{\mathbf{E}}-\overrightarrow{\mathbf{b}} \cdot \frac{\partial}{\partial b} \overrightarrow{\mathbf{E}},
\end{aligned}
$$

which implies that

$$
\mathbf{E}_{n}^{n}-\mathbf{E}_{b}^{b}=-\mathbf{E}^{n} \operatorname{div} \overrightarrow{\mathbf{n}}+\mathbf{E}^{b} d i v \overrightarrow{\mathbf{b}}
$$

From Eqs. $(15,28,43,48)$, we compute that

$$
\begin{aligned}
& 0=\nabla \cdot \overrightarrow{\mathbf{B}}=\left(\overrightarrow{\mathbf{t}} \frac{\partial}{\partial s}+\overrightarrow{\mathbf{n}} \frac{\partial}{\partial n}-\overrightarrow{\mathbf{b}} \frac{\partial}{\partial b}\right) \cdot \overrightarrow{\mathbf{B}}, \\
& 0=\overrightarrow{\mathbf{t}} \cdot \frac{\partial}{\partial s} \overrightarrow{\mathbf{B}}+\overrightarrow{\mathbf{n}} \cdot \frac{\partial}{\partial n} \overrightarrow{\mathbf{B}}+\overrightarrow{\mathbf{b}} \cdot \frac{\partial}{\partial b} \overrightarrow{\mathbf{B}},
\end{aligned}
$$

which implies that

$$
\mathbf{E}_{n}^{b}-\mathbf{E}_{b}^{n}=\mathbf{E}^{n} \operatorname{div} \overrightarrow{\mathbf{b}}-\mathbf{E}^{b} \operatorname{div} \overrightarrow{\mathbf{n}} .
$$

If we further consider Eqs. $(51,52)$, then it is obtained Laplacian-like formal equations along with the $n$-lines and $b-$ lines of the electromagnetic waves as follows

$$
\begin{aligned}
& \frac{\partial^{2}}{\partial n^{2}} \mathbf{E}^{b}-\frac{\partial^{2}}{\partial b^{2}} \mathbf{E}^{b}=\mathbf{E}^{n}\left((\operatorname{div} \overrightarrow{\mathbf{b}})_{n}-(\operatorname{div} \overrightarrow{\mathbf{n}})_{b}\right) \\
&+\mathbf{E}^{b}\left((\operatorname{div} \overrightarrow{\mathbf{b}})_{b}-(\operatorname{div} \overrightarrow{\mathbf{n}})_{n}\right) \\
&+\operatorname{div} \overrightarrow{\mathbf{b}}\left(\mathbf{E}_{n}^{n}+\mathbf{E}_{b}^{b}\right)-\operatorname{div} \overrightarrow{\mathbf{n}}\left(\mathbf{E}_{n}^{b}+\mathbf{E}_{b}^{n}\right) \\
& \frac{\partial^{2}}{\partial n^{2}} \mathbf{E}^{n}-\frac{\partial^{2}}{\partial b^{2}} \mathbf{E}^{n}=\mathbf{E}^{n}\left((\operatorname{div} \overrightarrow{\mathbf{b}})_{b}-(\operatorname{div} \overrightarrow{\mathbf{n}})_{n}\right) \\
&+\mathbf{E}^{b}\left(-(\operatorname{div} \overrightarrow{\mathbf{b}})_{b}-(\operatorname{div} \overrightarrow{\mathbf{n}})_{n}\right) \\
&+\operatorname{div} \overrightarrow{\mathbf{b}}\left(\mathbf{E}_{b}^{n}+\mathbf{E}_{n}^{b}\right)-\operatorname{div} \overrightarrow{\mathbf{n}}\left(\mathbf{E}_{n}^{n}+\mathbf{E}_{b}^{b}\right)
\end{aligned}
$$

Detailed discussion on the exact solutions of this Laplacianlike formal equations will be presented later in the application section. From Eqs. $(14,25,40,49)$, we also compute that

$$
\begin{aligned}
& \epsilon v \frac{\partial \overrightarrow{\mathbf{E}}}{\partial u}=\nabla \times \overrightarrow{\mathbf{B}}=\left(\overrightarrow{\mathbf{t}} \frac{\partial}{\partial s}+\overrightarrow{\mathbf{n}} \frac{\partial}{\partial n}-\overrightarrow{\mathbf{b}} \frac{\partial}{\partial b}\right) \times \overrightarrow{\mathbf{B}}, \\
& \epsilon v \frac{\partial \overrightarrow{\mathbf{E}}}{\partial u}=\overrightarrow{\mathbf{t}} \times \frac{\partial}{\partial s} \overrightarrow{\mathbf{B}}+\overrightarrow{\mathbf{n}} \times \frac{\partial}{\partial n} \overrightarrow{\mathbf{B}}-\overrightarrow{\mathbf{b}} \times \frac{\partial}{\partial b} \overrightarrow{\mathbf{B}},
\end{aligned}
$$

which implies that

$$
\begin{aligned}
\epsilon v \frac{\partial \overrightarrow{\mathbf{E}}}{\partial u} & =\left(-\mathbf{E}_{n}^{n}+\mathbf{E}^{b} d i v \overrightarrow{\mathbf{b}}-\mathbf{E}_{b}^{b}+\mathbf{E}^{n}(\kappa+\operatorname{div} \overrightarrow{\mathbf{n}})\right) \overrightarrow{\mathbf{t}} \\
& +\left(\mathbf{E}_{s}^{n}-\pi_{n} \mathbf{E}^{b}-\delta_{b s} \mathbf{E}^{n}\right) \overrightarrow{\mathbf{n}} \\
& +\left(-\mathbf{E}_{s}^{b}+\pi_{b} \mathbf{E}^{n}-\delta_{n s} \mathbf{E}^{b}\right) \overrightarrow{\mathbf{b}}
\end{aligned}
$$

where it is assumed for the rest of the paper that $\epsilon v=1$ for the simple reason. From Eqs. $(15,28,43,50)$,we finally compute that

$$
\begin{aligned}
-\frac{\partial \overrightarrow{\mathbf{B}}}{\partial u} & =\nabla \times \overrightarrow{\mathbf{E}}=\left(\overrightarrow{\mathbf{t}} \frac{\partial}{\partial s}+\overrightarrow{\mathbf{n}} \frac{\partial}{\partial n}-\overrightarrow{\mathbf{b}} \frac{\partial}{\partial b}\right) \times \overrightarrow{\mathbf{E}} \\
-\frac{\partial \overrightarrow{\mathbf{B}}}{\partial u} & =\overrightarrow{\mathbf{t}} \times \frac{\partial}{\partial s} \overrightarrow{\mathbf{E}}+\overrightarrow{\mathbf{n}} \times \frac{\partial}{\partial n} \overrightarrow{\mathbf{E}}-\overrightarrow{\mathbf{b}} \times \frac{\partial}{\partial b} \overrightarrow{\mathbf{E}}
\end{aligned}
$$

which implies that

$$
\begin{aligned}
\frac{\partial \overrightarrow{\mathbf{B}}}{\partial u} & =\left(\mathbf{E}_{n}^{b}-\mathbf{E}^{n} d i v \overrightarrow{\mathbf{b}}+\mathbf{E}_{b}^{n}-\mathbf{E}^{b}(\kappa+\operatorname{div} \overrightarrow{\mathbf{n}})\right) \overrightarrow{\mathbf{t}} \\
& +\left(-\mathbf{E}_{s}^{b}+\pi_{n} \mathbf{E}^{n}+\delta_{b s} \mathbf{E}^{b}\right) \overrightarrow{\mathbf{n}} \\
& +\left(\mathbf{E}_{s}^{n}-\pi_{b} \mathbf{E}^{b}+\delta_{n s} \mathbf{E}^{n}\right) \overrightarrow{\mathbf{b}}
\end{aligned}
$$

Here we should recall that $(s, n, b)$ and $u$ are space and time variables, respectively. These subscripts also denote partial derivatives. Nonlinear partial differential equation systems are given by Eqs. (51-54) and provide to investigate the significant connections between the geometry and nonlinear evolution of the given mechanism. Equations $(55,56)$ represent the geometrically observable time evolution systems of electric field and magnetic field vectors along the optical fiber governed by the Maxwellian equations. Characterizations of the time evolution of the unit Frenet-Serret vectors $(\overrightarrow{\mathbf{t}}, \overrightarrow{\mathbf{n}}, \overrightarrow{\mathbf{b}})$ and other geometric quantities will be the main subject of the next section. Even though we use the Maxwellian equation to describe the evolution of the electromagnetic wave along with the fiber, our investigation can easily be applied for quantum wave equations. In this context, this study will lead to a positive and direct impact on the research of the evolution of quantum particles.

\section{Maxwellian motion of Frenet-Serret vectors along with the optical fiber in Minkowski space}

The research of the evolution of a space curve has productive applications in many branches of science. Equations of moving curves have been analyzed by using the representation of Frenet-Serret orthonormal vectors and compatibility equations on these vectors by many researchers. These equations are mostly expressed by non-linear partial differential equations. Their main components are torsion and curvature functions of the curve. However, these representations of the moving curve are generally a challenging task. Here, we define a new class of evolutions by considering the Maxwell's equation of the propagated electromagnetic waves along with 
the fiber in Minkowski space. Thus we aim to improve a novel and special class of evolution kinematics.

Since $(\overrightarrow{\mathbf{t}}, \overrightarrow{\mathbf{n}}, \overrightarrow{\mathbf{b}})$ is an orthonormal unit triad, it is canonically true that $\overrightarrow{\mathbf{t}} \cdot \overrightarrow{\mathbf{t}}=1$, which implies $\overrightarrow{\mathbf{t}_{u}} \cdot \overrightarrow{\mathbf{t}}=0$. So we may assume that

$$
\overrightarrow{\mathbf{t}_{u}}=a_{1} \overrightarrow{\mathbf{n}}+a_{2} \overrightarrow{\mathbf{b}}
$$

We also know from the assumption that $\overrightarrow{\mathbf{E}} \cdot \overrightarrow{\mathbf{t}}=0$, which implies $\overrightarrow{\mathbf{E}}_{u} \cdot \overrightarrow{\mathbf{t}}=-\overrightarrow{\mathbf{E}} \cdot \overrightarrow{\mathbf{t}_{u}}$. So it is obtained that

$$
\begin{aligned}
a_{1} \mathbf{E}^{n}+a_{2} \mathbf{E}^{b} & =-\left(-\mathbf{E}_{n}^{n}+\mathbf{E}^{b} d i v \overrightarrow{\mathbf{b}}\right. \\
& \left.-\mathbf{E}_{b}^{b}+\kappa \mathbf{E}^{n}+\mathbf{E}^{n} \operatorname{div} \overrightarrow{\mathbf{n}}\right) .
\end{aligned}
$$

Now, if we consider the fact that $\overrightarrow{\mathbf{B}}=\overrightarrow{\mathbf{t}} \times \overrightarrow{\mathbf{E}}$, then we obtain the Maxwellian evolution of electromagnetic fields in the following manner

$$
\overrightarrow{\mathbf{B}}_{u}=\overrightarrow{\mathbf{t}_{u}} \times \overrightarrow{\mathbf{E}}+\overrightarrow{\mathbf{t}} \times \overrightarrow{\mathbf{E}}_{u}
$$

Hence, from Eqs. $(55-57)$, we obtain that

$$
\begin{aligned}
a_{1} & =\frac{1}{\Omega}\left(\frac{\partial \rho}{\partial b}\left(\left(\mathbf{E}^{n}\right)^{2}+\left(\mathbf{E}^{b}\right)^{2}\right)\right. \\
& \left.-\mathbf{E}^{n}\left(\mathbf{E}_{n}^{n}+\mathbf{E}_{b}^{b}\right)-\mathbf{E}^{b}\left(\mathbf{E}_{n}^{b}+\mathbf{E}_{b}^{n}\right)+2 \frac{\partial \rho}{\partial n}\right), \\
a_{2} & =-\frac{1}{\Omega}\left(\frac{\partial \rho}{\partial n}\left(\left(\mathbf{E}^{n}\right)^{2}+\left(\mathbf{E}^{b}\right)^{2}\right)\right. \\
& \left.-\mathbf{E}^{b}\left(\mathbf{E}_{n}^{n}+\mathbf{E}_{b}^{b}\right)-\mathbf{E}^{n}\left(\mathbf{E}_{n}^{b}+\mathbf{E}_{b}^{n}\right)+2 \frac{\partial \rho}{\partial b}\right),
\end{aligned}
$$

where $(\partial / \partial b) \rho=(\kappa+\operatorname{div} \overrightarrow{\mathbf{n}}),(\partial / \partial n) \rho=\operatorname{div} \overrightarrow{\mathbf{b}}, \Omega=$ $\left(\mathbf{E}^{b}\right)^{2}-\left(\mathbf{E}^{n}\right)^{2}(\Omega \neq 0)$. Here, we also obtain the following identity

$$
\frac{\mathbf{E}_{s}^{n}+\mathbf{E}_{s}^{b}}{\mathbf{E}^{n}+\mathbf{E}^{b}}=\frac{\pi_{b}-\pi_{n}-\delta_{n s}+\delta_{b s}}{2}
$$

where $\mathbf{E}^{n}+\mathbf{E}^{b} \neq 0$. To sum up, in Minkowski space, the Maxwellian evolution of the tangent vector is stated by

$$
\begin{aligned}
\overrightarrow{\mathbf{t}_{u}} & =\frac{1}{\Omega}\left(\frac{\partial \rho}{\partial b}\left(\left(\mathbf{E}^{n}\right)^{2}+\left(\mathbf{E}^{b}\right)^{2}\right)-\mathbf{E}^{n}\left(\mathbf{E}_{n}^{n}+\mathbf{E}_{b}^{b}\right)\right. \\
& \left.-\mathbf{E}^{b}\left(\mathbf{E}_{n}^{b}+\mathbf{E}_{b}^{n}\right)+2 \frac{\partial \rho}{\partial n}\right) \overrightarrow{\mathbf{n}}-\frac{1}{\Omega}\left(\frac{\partial \rho}{\partial n}\left(\left(\mathbf{E}^{n}\right)^{2}+\left(\mathbf{E}^{b}\right)^{2}\right)\right. \\
& \left.-\mathbf{E}^{b}\left(\mathbf{E}_{n}^{n}+\mathbf{E}_{b}^{b}\right)-\mathbf{E}^{n}\left(\mathbf{E}_{n}^{b}+\mathbf{E}_{b}^{n}\right)+2 \frac{\partial \rho}{\partial b}\right) \overrightarrow{\mathbf{b}}
\end{aligned}
$$

Recalling that $\overrightarrow{\mathbf{t}} \cdot \overrightarrow{\mathbf{n}}=0$ and $\overrightarrow{\mathbf{t}} \cdot \overrightarrow{\mathbf{b}}=0$, we compute the Maxwellian evolution of the normal and binormal vectors in the following manner

$$
\begin{aligned}
\overrightarrow{\mathbf{n}_{u}} & =-\frac{1}{\Omega}\left(\frac{\partial \rho}{\partial b}\left(\left(\mathbf{E}^{n}\right)^{2}+\left(\mathbf{E}^{b}\right)^{2}\right)-\mathbf{E}^{n}\left(\mathbf{E}_{n}^{n}+\mathbf{E}_{b}^{b}\right)\right. \\
& \left.-\mathbf{E}^{b}\left(\mathbf{E}_{n}^{b}+\mathbf{E}_{b}^{n}\right)+2 \frac{\partial \rho}{\partial n}\right) \overrightarrow{\mathbf{t}}+\Upsilon \overrightarrow{\mathbf{b}} \\
\overrightarrow{\mathbf{b}_{u}} & =-\frac{1}{\Omega}\left(\frac{\partial \rho}{\partial n}\left(\left(\mathbf{E}^{n}\right)^{2}+\left(\mathbf{E}^{b}\right)^{2}\right)-\mathbf{E}^{b}\left(\mathbf{E}_{n}^{n}+\mathbf{E}_{b}^{b}\right)\right. \\
& \left.-\mathbf{E}^{n}\left(\mathbf{E}_{n}^{b}+\mathbf{E}_{b}^{n}\right)+2 \frac{\partial \rho}{\partial b}\right) \overrightarrow{\mathbf{t}}-\Upsilon \overrightarrow{\mathbf{n}},
\end{aligned}
$$

where $\Upsilon$ is an arbitrarily smooth function. Consequently, we have the corresponding formula of the Maxwellian evolution of the Frenet-Serret vectors in Minkowski space

$$
\frac{\partial}{\partial u}\left(\begin{array}{c}
\overrightarrow{\mathbf{t}} \\
\overrightarrow{\mathbf{n}} \\
\overrightarrow{\mathbf{b}}
\end{array}\right)=\left(\begin{array}{ccc}
0 & r & q \\
-r & 0 & \Upsilon \\
q & \Upsilon & 0
\end{array}\right)\left(\begin{array}{c}
\overrightarrow{\mathbf{t}} \\
\overrightarrow{\mathbf{n}} \\
\overrightarrow{\mathbf{b}}
\end{array}\right),
$$

where

$$
\begin{aligned}
r & =\frac{1}{\Omega}\left(\frac{\partial \rho}{\partial b}\left(\left(\mathbf{E}^{n}\right)^{2}+\left(\mathbf{E}^{b}\right)^{2}\right)-\mathbf{E}^{n}\left(\mathbf{E}_{n}^{n}+\mathbf{E}_{b}^{b}\right)\right. \\
& \left.-\mathbf{E}^{b}\left(\mathbf{E}_{n}^{b}+\mathbf{E}_{b}^{n}\right)+2 \frac{\partial \rho}{\partial n}\right), \\
q & =-\frac{1}{\Omega}\left(\frac{\partial \rho}{\partial n}\left(\left(\mathbf{E}^{n}\right)^{2}+\left(\mathbf{E}^{b}\right)^{2}\right)-\mathbf{E}^{b}\left(\mathbf{E}_{n}^{n}+\mathbf{E}_{b}^{b}\right)\right. \\
& \left.-\mathbf{E}^{n}\left(\mathbf{E}_{n}^{b}+\mathbf{E}_{b}^{n}\right)+2 \frac{\partial \rho}{\partial b}\right) .
\end{aligned}
$$

Here the matrix is not an antisymmetric form as expected due to the characterization of the unit vector fields in Minkowski space. This characterization is determined by the inner product of unit vector fields given earlier as follows

$$
\overrightarrow{\mathbf{t}} \cdot \overrightarrow{\mathbf{t}}=1, \overrightarrow{\mathbf{n}} \cdot \overrightarrow{\mathbf{n}}=1, \overrightarrow{\mathbf{b}} \cdot \overrightarrow{\mathbf{b}}=-1
$$

More details about the consequences of this characterization can be found in [18]. If we assume that compatibility conditions hold and consider the above characterization, then the Maxwellian evolution of all geometric quantities given by Eqs. (5-13) and the arbitrary function $\Upsilon$ is given by

$$
\begin{aligned}
\kappa_{u} & =r_{s}+q \tau, \tau_{u}=\kappa q, \Upsilon=0, \\
\left(\delta_{n s}\right)_{u} & =r_{n}-q \operatorname{div} \overrightarrow{\mathbf{b}} \\
\left(\pi_{b}-\tau\right)_{u} & =-q_{n}-r \operatorname{div} \overrightarrow{\mathbf{b}} \\
(\operatorname{div} \overrightarrow{\mathbf{b}})_{u} & =r\left(\pi_{b}-\tau\right)+q \delta_{n s} \\
\left(\delta_{b s}\right)_{u} & =-q_{b}-r(\kappa+\operatorname{div} \overrightarrow{\mathbf{n}}) \\
\left(\pi_{n}-\tau\right)_{u} & =-r_{b}-q(\kappa+\operatorname{div} \overrightarrow{\mathbf{n}}) \\
(\kappa+\operatorname{div} \overrightarrow{\mathbf{n}})_{u} & =r \delta_{b s}-q\left(\pi_{n}-\tau\right)
\end{aligned}
$$


The above system may be considered as Mainardi-GaussCodazzi equations for surfaces parametrized with anholonomic coordinates. For a given solution of this system, the evolution equations presented by $(1-3,62)$ are compatible and may define a surface up to their position in space.

\section{Application: Soliton solutions of Laplacian- Like formalism of electromagnetic waves along with the uniform optical fiber}

The research of the traveling wave transformation of nonlinear evolution equations (NLEE) plays an important role to examine the internal mechanism of sophisticated nonlinear physical phenomena. Most of the physical phenomena, including plasma physics, quantum mechanics, fluid mechanics, propagation of shallow-water waves, chemical kinematics, optical fibers, electricity, and magnetism are modeled by NLEEs. The presence of wave solution displays a considerably higher rate of recurrence in nature. However, nonlinear cases are challenging, and they are not easy to control since the nonlinearity of the mechanisms may cause an unexpected change of the systems. Therefore, advanced nonlinear techniques have been developed by many authors to compute the exact solutions of NLEEs.

In this section, we recall the fundamental steps of the traveling wave hypothesis approach. Thus we aim to obtain exact traveling wave solutions of the Laplacian-like formal equations given by Eqs. $(53,54)$ for some special cincumstances. We also provide numerical simulations to support analytic outcomes.

For simplicity, let assume that electromagnetic waves along the uniform optical fiber satisfy the following nonlinear evolution equation system induced by the Eqs. $(53,54)$

$$
\begin{gathered}
\frac{\partial^{2} \mathbf{E}^{n}(n, b)}{\partial n^{2}}-\frac{\partial^{2} \mathbf{E}^{n}(n, b)}{\partial b^{2}}=\mathbf{E}^{n}(n, b)+\mathbf{E}^{b}(n, b), \\
\frac{\partial^{2} \mathbf{E}^{b}(n, b)}{\partial n^{2}}-\frac{\partial^{2} \mathbf{E}^{b}(n, b)}{\partial b^{2}}=\mathbf{E}^{n}(n, b)-\mathbf{E}^{b}(n, b) .
\end{gathered}
$$

We consider the given below traveling wave transformation for the Eq. (63)

$$
\begin{aligned}
\mathbf{E}^{n}(n, b) & =w_{1}(\phi), \quad \mathbf{E}^{b}(n, b)=w_{2}(\phi), \\
\phi & =n-Q b,
\end{aligned}
$$

where $Q$ describes the speed of the wave. If we plug the Eq. (64) into the Eq. (63) then it is obtained that

$$
\begin{aligned}
\frac{1}{\gamma^{2}} w_{1}^{\prime \prime}(\phi)-w_{1}(\phi)-w_{2}(\phi) & =0, \\
\frac{1}{\gamma^{2}} w_{2}^{\prime \prime}(\phi)-w_{1}(\phi)+w_{2}(\phi) & =0 .
\end{aligned}
$$

where $\gamma=1 / \sqrt{1-Q^{2}}$. If we further solve the Eq. (65) by the Mathematica, we compute that

$$
\begin{aligned}
w_{1} & =A_{1}(\gamma) e^{i \phi_{\gamma}}+B_{1}(\gamma) e^{-i \phi_{\gamma}} \\
& +C_{1}(\gamma) e^{\phi_{\gamma}}+D_{1}(\gamma) e^{-\phi_{\gamma}}
\end{aligned}
$$

where

$$
\begin{aligned}
& A_{1}(\gamma)=-\frac{2^{\frac{1}{4}}}{8}\left[2^{\frac{1}{4}}\left(c_{3}-c_{1,-}\right)-i \frac{\left(c_{4}-c_{2,-}\right)}{\gamma}\right], \\
& B_{1}(\gamma)=-\frac{2^{\frac{1}{4}}}{8}\left[2^{\frac{1}{4}}\left(c_{3}-c_{1,-}\right)+i \frac{\left(c_{4}-c_{2,-}\right)}{\gamma}\right], \\
& C_{1}(\gamma)=\frac{2^{\frac{1}{4}}}{8}\left[2^{\frac{1}{4}}\left(c_{3}+c_{1,+}\right)+\frac{\left(c_{4}+c_{2,+}\right)}{\gamma}\right], \\
& D_{1}(\gamma)=\frac{2^{\frac{1}{4}}}{8}\left[2^{\frac{1}{4}}\left(c_{3}+c_{1,+}\right)-\frac{\left(c_{4}+c_{2,+}\right)}{\gamma}\right]
\end{aligned}
$$

and

$$
\begin{aligned}
w_{2} & =A_{2}(\gamma) e^{i \phi_{\gamma}}+B_{2}(\gamma) e^{-i \phi_{\gamma}} \\
& +C_{2}(\gamma) e^{\phi_{\gamma}}+D_{2}(\gamma) e^{-\phi_{\gamma}},
\end{aligned}
$$

where

$$
\begin{aligned}
& A_{2}(\gamma)=-\frac{2^{\frac{1}{4}}}{8}\left[2^{\frac{1}{4}}\left(c_{1}-c_{3,+}\right)-i \frac{\left(c_{2}-c_{4,+}\right)}{\gamma}\right], \\
& B_{2}(\gamma)=-\frac{2^{\frac{1}{4}}}{8}\left[2^{\frac{1}{4}}\left(c_{1}-c_{3,+}\right)+i \frac{\left(c_{2}-c_{4,+}\right)}{\gamma}\right], \\
& C_{2}(\gamma)=\frac{2^{\frac{1}{4}}}{8}\left[2^{\frac{1}{4}}\left(c_{1}+c_{3,-}\right)+\frac{\left(c_{2}+c_{4,+}\right)}{\gamma}\right], \\
& D_{2}(\gamma)=\frac{2^{\frac{1}{4}}}{8}\left[2^{\frac{1}{4}}\left(c_{1}+c_{3,-}\right)-\frac{\left(c_{2}+c_{4,+}\right)}{\gamma}\right] .
\end{aligned}
$$

Here it is also considered that

$$
c_{i, \pm}=c_{i}(\sqrt{2} \pm 1) \text { and } \phi_{\gamma}=2^{\frac{1}{4}} \gamma \phi .
$$

\section{Conclusion}

We have examined the geometric evolution of the electromagnetic waves carried by the light propagating along with the uniform optical fiber in Minkowski space. We firstly recall a classical method to find the geometric phase of the propagated light along with the fiber in Minkowski space. By using this straightforward approach, we define two novel geometric phases associated with the evolution of the polarization vectors in the normal and binormal directions along with the optical fiber. We also give their connections with parallel transportation laws in Minkowski space. Then we consider evolution equations of the electric field and magnetic field vectors along the optical fiber governed by the Maxwellian equations. Hence we obtain formal definitions of the time evolution of the unit Frenet-Serret vectors $(\overrightarrow{\mathbf{t}}, \overrightarrow{\mathbf{n}}, \overrightarrow{\mathbf{b}})$ and associated geometric quantities. Once the evolution equations of quantum or non-quantum systems are described one knows that many interesting soliton equations can be related 
to these evolution systems. This fact also holds not only in the ordinary space but also in other spacetime structures, including ordinary space, De-Sitter space, anti De-Sitter space, etc. For further research, we aim to connect Maxwellian evolution equations with well-known completely integrable equations and their soliton solutions. The above-mentioned observations and obtained results propose possible applications and new research areas also in pure geometric research such as inextensibility conditions of Maxwellian evolution equations, creating Maxwellian envelope surface, etc.

\section{Acknowledgments}

We thank the anonymous reviewers for their careful reading of our manuscript and their many insightful comments and suggestions.
1. A.M. Smith, Appl. Opt. 17 (1978) 52. https://doi.org/ $10.1364 / \mathrm{AO} .17 .000052$

2. J.N. Ross, Opt. Quantum Electron. 16 (1984) 455. https: //doi.org/10.1007/BF00619638

3. A. Tomita, Y. Chiao, Phys. Rev. Lett. 57 (1986) 937. https: //doi.org/10.1103/PhysRevLett.57.937

4. R.Y. Chiao, Y.S. Wu, Phys. Rev. Lett. 57 (1986) 933. https : //doi.org/10.1103/PhysRevLett.57.933

5. F.D.M. Haldane, Optics Lett. 11 (1986) 730. https : // doi. org/10.1364/OL.11.000730

6. T. Körpınar, R.C. Demirkol, J. Mod. Optics. 66 (2019) 857.

7. T. Körpınar, R.C. Demirkol, Z. Körpınar, Eur. Phys. J. D. 73 (2019) 203. https://doi.org/10.1140/epjd/ e2019-100250-7

8. T. Körpınar, R.C. Demirkol, Z. Körpınar, Int. J. Geom. Methods M. 16 (2019) 1950117.

9. T. Körpınar, R.C. Demirkol, Z. Körpınar, Rev. Mex. Fis. 65 (2019) 626.

10. T. Körpınar, R.C. Demirkol, Optik. 200 (2020) 163334. https://doi.org/10.1016/j.ijleo.2020. 164561
11. R. Betchov, J. Fluid Mech. 22 (1965) 471. https://doi. org/10.1017/S0022112065000915

12. L.D. Rios, Rend. Circ. Mat. Palermo. 22 (1906) 117. https: //doi.org/10.1007/BF03018608

13. W. Schief, Phys. Plasmas. 10 (2003) 2677. https://doi. org/10.1063/1.1577347

14. W.K. Schief, C. Rogers, J. Geom. Phys. 54 (2005) 286. https://doi.org/10.1016/j.geomphys.2004. 10.001

15. H. Hasimoto, J. Fluid Mech. 51 (1972) 293. https://doi. org/10.1017/S0022112072002307

16. E.M. Frins, W. Dultz, J. Lightwave Technol. 15 (1997) 144. https://doi.org/10.1109/50.552122

17. O. Yamashita, Opt. Commun. 285 (2012) 3061. https:// doi.org/10.1016/j.optcom.2012.05.013

18. R. Lopez, Int. Electron. J. Geom. 7 (2014) 44. https:// doi.org/10.36890/iejg.594497 